\title{
Dawn of a New Era
}

\section{- The Far Lower, the Far Better Low-Density Lipoprotein Cholesterol Story in Japan -}

Tatsuro Ishida, MD, $\mathrm{PhD}$

$\mathbf{L}$ ow-density lipoprotein cholesterol (LDL-C) is a wellestablished causative risk factor for coronary artery disease (CAD), and intensive LDL-lowering treatment with statins has efficiently reduced coronary events and deaths. ${ }^{1}$ Nevertheless, many patients in Japan with high coronary risk have not achieved LDL-C target goals. ${ }^{2}$ Furthermore, clinical trials suggest that residual risks remain for $\mathrm{CAD}$ even with maximal potency statins. ${ }^{3}$ Additional reduction in LDL-C, to $<60 \mathrm{mg} / \mathrm{dl}$, using the non-statin drug, ezetimibe, has been shown in recent years to result in a further reduction of cardiovascular events. ${ }^{4}$ However, as it has already been 30 years after the introduction of statins and 10 years after ezetimibe came onto the market, it is time for the next breakthrough.

\section{Article p 1980}

The proprotein convertase subtilisin/kexin type 9 (PCSK9) is known to increase LDL-C levels through interference with normal physiological recycling of hepatic LDL receptors (Figure); it binds to the cell surface LDL receptor, and is taken up together into the cytoplasm, and thereby promotes the degradation of the LDL receptor in the lysosome. Statins reduce plasma LDL-C levels through inhibition of cholesterol biosynthesis and by upregulation of LDL receptors. In addition, statins increase the expression of PCSK9, which results in attenuation of the LDL-lowering effect. Monoclonal antibodies against PCSK9 have been developed and shown to significantly reduce LDL-C levels in a large number of phase 2 and 3 studies mainly performed in Western countries. ${ }^{5}$

The prevalence of CAD in Japan is known to be significantly lower than in Western countries, despite decades of westernized lifestyle and comparable plasma LDL-C levels. Moreover, in Japan where statins are usually used at half to one-quarter of the doses used in Western countries, they are still found to have benefits. Thus, the efficacy of LDL-lowering therapy with PCSK9 inhibitors should be validated in Japanese subjects. Two PCSK9 inhibitors, alirocumab and evolocumab, recently became available in Japan. ${ }^{6-8}$ ODYSSEY Japan is a randomized, double-blinded clinical trial that validated the efficacy and safety of alirocumab in Japanese patients with heterozygous familial hypercholesterolemia $(\mathrm{FH})$ or non- $\mathrm{FH}$ with high coronary risks. In this issue of the Journal, Teramoto and colleagues ${ }^{9}$ compare the effect of alirocumab $(75$ or $150 \mathrm{mg}$ every 2 weeks) and placebo for 52 weeks in patients on stable statin therapy. Alirocumab reduced the LDL-C levels by more than $60 \%$, to 53.5 and $53.4 \mathrm{mg} / \mathrm{dl}$ at 24 and 52 weeks, respectively. The LDL-lowering effect was sustained to the endpoint of week 52, and no rebound effect was observed. Also, alirocumab markedly reduced non-HDL cholesterol and apolipoprotein B levels. In some cases, the LDL-C level was

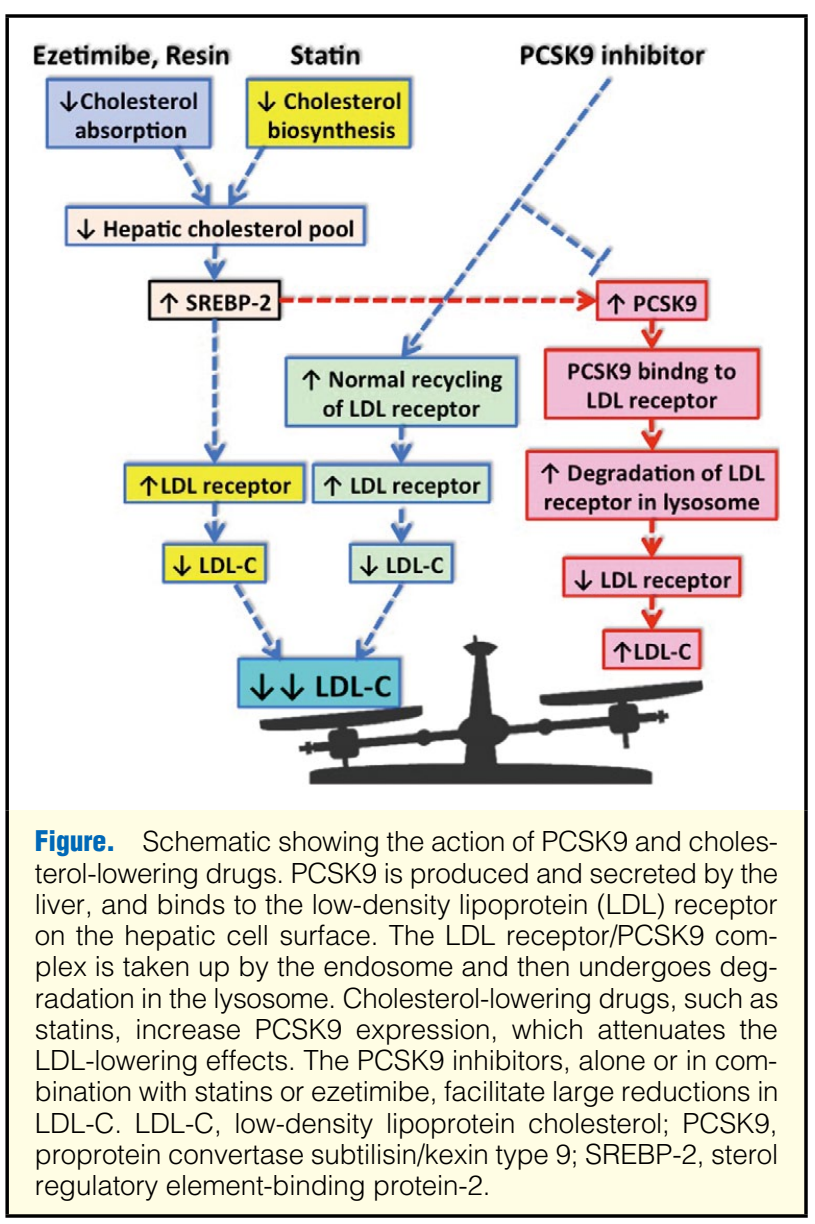

The opinions expressed in this article are not necessarily those of the editors or of the Japanese Circulation Society.

Received July 26, 2016; accepted July 27, 2016; released online August 4, 2016

Division of Cardiovascular Medicine, Kobe University Graduate School of Medicine, Kobe, Japan

Mailing address: Tatsuro Ishida, MD, PhD, Division of Cardiovascular Medicine, Kobe University Graduate School of Medicine, 7-5-1

Kusunoki-cho, Chuo-ku, Kobe 650-0017, Japan. E-mail: ishida@med.kobe-u.ac.jp

ISSN-1346-9843 doi:10.1253/circj.CJ-16-0747

All rights are reserved to the Japanese Circulation Society. For permissions, please e-mail: cj@j-circ.or.jp 
Table. Possible Current Clinical Applications of PCSK9 Inhibitors*

High-risk patients not achieving LDL-C target goals with highdose statins

Several types of familial hypercholesterolemia**

Statin intolerance

High lipoprotein(a)

Others

*These applications do not always come under insurance coverage in Japan. ${ }^{\star *}$ Effect has not been seen in homozygous patients with dual allele LDL receptor negative mutants or in those who are homozygous for autosomal recessive hypercholesterolemia. LDL-C, low-density lipoprotein cholesterol.

$<25 \mathrm{mg} / \mathrm{dl}$, but no significant adverse effect was seen. The agent was generally well tolerated and few patients discontinued treatment because of adverse effects, indicating that it does not appear to have the hepatic and muscle-related side effects often accompanying statin therapy in Japanese patients.

It has been postulated that statins have a variety of antiatherosclerotic effects beyond the LDL-C level, which are referred to as "pleiotropic effects". It remains unclear whether the LDL reduction by the PCSK9 inhibitor is accompanied by a comparable event reduction to that of statins. Cardiovascular protective effects of PCSK9 inhibitors have not been definitely established yet, though some analyses of incidentally reported or secondary outcomes have suggested potential beneficial effects. It has been reported that gain-of-function or loss-of-function mutations in the PCSK9 genetic locus are associated with high or low incidence of atherosclerosis, respectively, which may support the benefit of PCSK9 inhibitors. It should be noted that there was a criticism that underutilization of the maximum dose of statins in high-risk patients may have overestimated the benefit of PCSK9 inhibition in some clinical trials. ${ }^{10}$ Thus, given the high cost of this drug, it seems reasonable at present to start this drug in high-risk patients on sufficient statin therapy who have not reached LDL-C target goals (Table). In addition, patients with statin intolerance or adverse effects may be good indications. ${ }^{11}$ It would also be clinically applicable for patients with several types of $\mathrm{FH}$, although it is not effective in patients who are homozygous for dual allele LDL-receptor negative mutants or those with homozygous autosomal recessive hypercholesterolemia. On the other hand, PCSK9 is a regulator of the plasma lipoprotein(a) concentration, which is a strong risk factor for CAD. ${ }^{12}$ Teramoto et al reported that alirocumab reduced lipoprotein(a) by $40 \%$, implicating the potential effect of this drug on the plasma lipoprotein(a) level. ${ }^{9}$ Furthermore, PCSK9 has been reported to promote overproduction of triglyceriderich, apolipoprotein B48- and B100-containing lipoproteins in the intestine and liver through both LDL receptor-dependent and -independent mechanisms, ${ }^{13}$ suggesting that PCSK9 inhibitors may reduce triglyceride-rich lipoproteins as well. The clinical effect of PCSK9 inhibitors on these non-LDL lipids and other primary hyperlipidemia should be investigated in the future.

In conclusion, the finding of a marked reduction of LDL-C without significant adverse effects well over 52 weeks may help this drug to expand the therapeutic strategy for hypercholesterolemia and high-risk patients on stable statin therapy. It has been believed by a large number of interventional trials of statins that "the lower the LDL-C, the better the cardiovascular outcome". However, it remains unclear if this is the case with an extremely low LDL-C level (ie, $<50-60 \mathrm{mg} / \mathrm{dl}$ ) in Japan. Now that the efficacy and safety of PCSK9 inhibitors are proven in Japanese patients, ${ }^{6-9}$ it is no exaggeration to say that we have reached a new era in determining whether "the far lower, the far better" story is true.

\section{Disclosures}

T.I. has received lecture honoraria from MSD and Mochida Pharmaceutical.

\section{References}

1. Baigent C, Blackwell L, Emberson J, Holland LE, Reith C, Bhala N, et al; Cholesterol Treatment Trialists' Collaboration. Efficacy and safety of more intensive lowering of LDL cholesterol: A metaanalysis of data from 170,000 participants in 26 randomized trials. Lancet 2010; 376: 1670-1681.

2. Teramoto T, Kashiwagi A, Ishibashi S, Daida H; Japan Lipid Guideline Achievement Program Investigators. Cross-sectional survey to assess the status of lipid management in high-risk patients with dyslipidemia: Clinical impact of combination therapy with ezetimibe. Curr Ther Res Clin Exp 2012; 73: 1-15.

3. Chapman MJ, Redfern JS, McGovern ME, Giral P. Niacin and fibrates in atherogenic dyslipidemia: Pharmacotherapy to reduce cardiovascular risk. Pharmacol Ther 2010; 126: 314-345.

4. Cannon CP, Blazing MA, Giugliano RP, McCagg A, White JA, Theroux P, et al. Ezetimibe added to statin therapy after acute coronary syndromes. N Engl J Med 2015; 372: 2387-2397.

5. McDonagh M, Peterson K, Holzhammer B, Fazio S. A systematic review of PCSK9 inhibitors alirocumab and evolocumab. J Manag Care Spec Pharm 2016; 22: 641-653q.

6. Teramoto T, Kobayashi M, Uno K, Takagi Y, Matsuoka O, Sugimoto $M$, et al. Efficacy and safety of alirocumab in Japanese subjects (Phase 1 and 2 Studies). Am J Cardiol 2016; 118: 56-63.

7. Kiyosue A, Honarpour N, Kurtz C, Xue A, Wasserman SM, Hirayama A. A phase 3 study of evolocumab (AMG 145) in statintreated Japanese patients at high cardiovascular risk. Am J Cardiol 2016; 117: 40-47.

8. Hirayama A, Honarpour N, Yoshida M, Yamashita S, Huang F, Wasserman SM, et al. Effects of evolocumab (AMG 145), a monoclonal antibody to PCSK9, in hypercholesterolemic, statin-treated Japanese patients at high cardiovascular risk-primary results from the phase 2 YUKAWA study. Circ J 2014; 78: 1073-1082.

9. Teramoto T, Kobayashi M, Tasaki H, Yagyu H, Higashikata T, Takagi Y, et al. Efficacy and safety of alirocumab in Japanese patients with heterozygous familial hypercholesterolemia or at high cardiovascular risk with hypercholesterolemia not adequately controlled with statins: ODYSSEY JAPAN randomized controlled trial. Circ J 2016; 80: 1980-1987.

10. Auer J, Berent R, Primus C. PCSK9 inhibitors and cardiovascular events. $N$ Engl J Med 2015; 373: 773.

11. Ogawa H, Matsui K, Saito Y, Sugiyama S, Jinnouchi H, Sugawara M, et al. Differences between rosuvastatin and atorvastatin in lipidlowering action and effect on glucose metabolism in Japanese hypercholesterolemic patients with concurrent diabetes: Lipid-lowering with highly potent statins in hyperlipidemia with type 2 diabetes patients (LISTEN) study. Circ J 2014; 78: 2512-2515.

12. Tada H, Kawashiri MA, Yoshida T, Teramoto R, Nohara A, Konno $\mathrm{T}$, et al. Lipoprotein(a) in familial hypercholesterolemia with proprotein convertase subtilisin/kexin type 9 gain-of-function mutations. Circ J 2016; 80: 512-518.

13. Rashid S, Tavori H, Brown PE, Linton MF, He J, Giunzioni I, et al. Proprotein convertase subtilisin kexin type 9 promotes intestinal overproduction of triglyceride-rich apolipoprotein B lipoproteins through both low-density lipoprotein receptor-dependent and -independent mechanisms. Circulation 2014; 130: $431-441$. 